\title{
Inspection of High Voltage Cables using X-ray Techniques
}

\author{
A P Robinson, P L Lewin, S J Sutton ${ }^{*}$ and S G Swingler \\ High Voltage Laboratory, University of Southampton, Hampshire, UK \\ "National Grid Transco, Warwickshire, UK \\ apr195@soton.ac.uk
}

\begin{abstract}
Defects that are accidentally introduced into high voltage cable joints during manufacture significantly decrease the working lifetime of the cable system. As a measure of quality assurance the joint can be non-destructively tested using $X$-rays to image the structure of the joint. The image produced can then be inspected for defects such as the thinning of the semiconducting sheaths or the insulation thickness. The location of the boundaries of these insulation components can be found by differentiating the profile of the $X$-ray image surface. Once these locations are known it is possible to calculate the thickness of these components. These thicknesses can then be used as a measure of quality assurance for the cable joint.
\end{abstract}

\section{INTRODUCTION}

The manufacture of high voltage cables using cross-linked polyethylene (XLPE) as insulation is an automated process that extrudes a layer of XLPE, sandwiched between two semiconducting sheathes, onto the central conductor. Using this method it is possible to ensure that the insulation is manufactured to a predetermined standard. The process, however, can only produce cables of a finite length due to transport restrictions. In order to produce longer lengths of cables, two or more cables are joined together. The jointing procedure, however, is a manual process due to its complexity. To determine the quality of the joint's insulation, the joint has to be non-destructively tested (NDT) to ensure that no voids, inclusions, mechanical damage, or delamination/reduction in thickness of the joint insulation/semiconducting sheaths have been accidentally created or introduced into the joint. The presence of any of these defects would have a detrimental effect on the working lifetime of the cable.

To NDT the cable joint for insulation defects, the internal structure of the joint is inspected using X-rays. The current inspection procedure involves the use of conventional X-ray and photographic film technologies. This process however suffers from problems that are associated with processing and storage of the photographic film. It is possible to replace the photographic plate with a charge coupled device (CCD) array optically coupled to a scintillating screen [1]. The image generated by the CCD camera can then be digitally inspected using image-processing algorithms, removing the problems associated with the processing and inspection of photographic plates. This paper describes the generation and inspection of digital NDT images of cable joints.

\section{SYSTEM HARDWARE}

The source used to generate the images was a Kevex E5014SMF X-ray source. This is a $50 \mathrm{kV} 1.0 \mathrm{~mA}$ dc tube with a focal spot size of $200 \times 400 \mu \mathrm{m}$. The camera used was an X-ray CCD camera built by Xcam Ltd scientific. This camera has $2048 \times 204813.5 \mu \mathrm{m}$ pixels with a 12-bit digitization level and a dynamic range of $33333: 1$, connected to a gadolinium oxysulphide scintillating fibre optic plate with a $1: 1$ ratio, producing a $27.6 \times 27.6 \mathrm{~mm}$ field of view.

\section{IMAGE PRODUCTION}

The joint inspected was a section of a $90 \mathrm{kV}$ submarine cable joint. Taped onto the outer semiconducting surface of this joint were three, $0.21 \mathrm{~mm}$ diameter ( $35 \mathrm{SWG}$ ) wires in an ' $\mathrm{H}$ ' configuration to provide a known defect. The joint section was imaged by irradiating it for 90 seconds with a tube voltage and current of $45 \mathrm{kV}$ and $0.7 \mathrm{~mA}$ respectively. The attenuated flux was then captured by the CCD array and converted to a 12-bit image.

In order to reduce the noise content in the image, 10 images of the same section of joint were generated. These images were then combined into one single image, $P$, by calculating the median value of the pixels on all 10 images, $P_{I}-P_{10}$, i.e.

$$
P_{(x, y)}=\operatorname{Median}\left(P_{1(x, y)}+P_{2(x, y)}+\ldots+P_{9(x, y)}+P_{10(x, y)}\right)
$$

Where $(x, y)$ is the current pixel location.

The median value was used, rather than the mean value, as it is resistant to individual pixel extremes [2]. The image was then smoothed to remove any static noise using a $3 \times 3$ median filter. This filter was used to remove any salt and pepper noise, while retaining image features [3].

Due to the field of view of the camera it was only possible to image small sections of the cable joint. The central conductor of the joint however is radiologically dense and cannot easily be imaged, therefore only the insulation and semiconductive layers can be inspected. The thickness of the layers for a $90 \mathrm{kV}$ cable joint is less than $27 \mathrm{~mm}$. It is possible, therefore, to image sections of the insulation and semiconductive layers either side of the conductor with a single image. The length of the joint section, however, is significantly greater than $27 \mathrm{~mm}$. To image the whole length of the joint section, the joint was 
mounted on a translational stage and indexed past the camera at $17 \mathrm{~mm}$ intervals. The $10 \mathrm{~mm}$ overlap of the images meant that any edge phenomena would not be present in the final image. This process can be repeated along the whole of the joint. For this paper the cable was only imaged three times.

Once the images were generated a montage image was constructed by joining the individual images at the centres of the overlaps. The centre of the overlap on each image was located $5 \mathrm{~mm}$ from the appropriate ends. This equates to 192 pixels from the edge of the image. This produced a $61 \mathrm{~mm}$ cross sectional image of joint insulation. This image is shown in Figure 2(d).

\section{IMAGE PROCESSING}

In order to ascertain the defect status of the joint section, the digital image has to be inspected for voids, inclusions, mechanical damage, and delamination/reduction in thickness of the joint insulation/semiconducting sheaths. This paper concentrates on the method of determining the thickness of the semiconducting sheaths and the insulation.

The general intensity profile of the joint image in Figure 2(d) is show in Figure 1 (a). In this image it can be seen that there are four main points of changes in the profile, caused by the material the X-ray is interacting with, and the thickness of that material. Referring to Figure 1, P1 is the interface between the air and the outer semiconducting sheath. The air to the left of the sheath has no significant affect on the X-ray and so

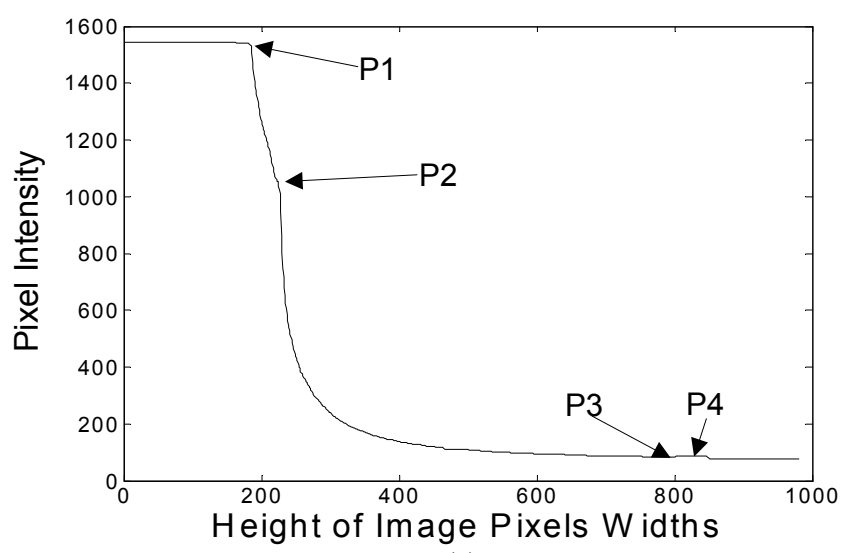

(a)

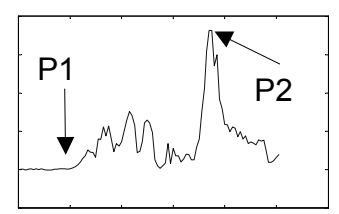

(b)

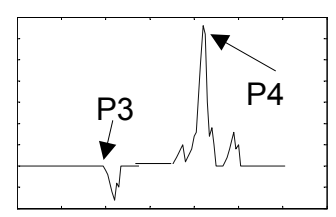

(c)
Figure 1 - The Four Image Points (a) Image Profile (b) Differentiated Image Profile showing P1 \& P2(c) Differentiated Image Profile showing P3 \& P4 only slight attenuation takes place resulting in the flat plane to the left of P1. From P1 to P2 the thickness of the semiconducting sheath interacting with the X-rays increases causing increased attenuation. This results in the gradual slope between $\mathrm{P} 1$ and $\mathrm{P} 2$. P2 is the interface between the outer sheath and the insulation. The joint insulation is more radiologically dense than the sheath and so the attenuation dramatically increases at this interface, causing the sudden change in pixel intensity. The curve profile from P2 to P3 is caused by the rate of change of the thickness of insulation from the outer semiconducting sheath to the inner semiconducting sheath. P3 is the interface between the insulation and the inner semiconducting sheath. As mentioned above the semiconducting sheath is less radiologically dense compared to the insulation and so the attenuation of the X-rays decreases between P3 and P4 resulting in the inflection in the intensity profile. P4 is the interface between the inner semiconducting sheath and the conductor. The conductor absorbs most of the X-rays irradiating it, which results in the decrease of the pixel intensities. If pixel row of P1-P4, in every pixel column of the image are found, the thickness of the insulation and semiconducting sheaths can be calculated by counting the number of pixels between these points and multiplying the number by the pixel size. From this it is then possible to automatically calculate the minimum, average and maximum thicknesses of the semiconducting sheaths and the insulation.

\section{Locating the Four Image Points}

If the image profile in Figure 1 (a) is differentiated it produces the profile shown in Figure $1(\mathrm{~b} \& \mathrm{c})$. To differentiate the image profile each pixel in every column of the image was discretely differentiated against the 'future' five pixels in the column, and the result averaged, i.e.

$$
P^{\prime}(x, y)=\frac{d P_{(x, y)}}{d y}=\frac{\left(\sum_{i}^{5} \frac{P_{(x, y)}-P_{(x, y+i)}}{i}\right)}{5}
$$

where $\mathrm{P}^{\prime}$ is the differentiated image profile

In the differentiated profile, P1 becomes the first significant upward deflection of the profile. P2 becomes the first local maximum in the profile. P3 becomes the point where the profile changes from positive to negative. P4 becomes the second local maximum in the profile. To find $\mathrm{P} 1$ in this profile it is necessary to find the first significant upward deflection. To find this point two characteristics of the surface are used. Firstly, to the left of P1 the image surface is relatively flat and secondly to the right of $\mathrm{P} 1$ there is a definite ramp in the profile. Due to the noise content in the image, however, the image surface to the left of P1 is not perfectly flat which means that there are small deflections in the 
differentiated surface to the left of P1, however either side of these deflections the profile is centred at zero. P1 therefore can be defined as the point where to its left the profile is generally flat and centred at zero, and to its right it is not flat and therefore not centred at zero. Mathematically this can be defined as $n$ 'previous' points have a modal value of zero and $\mathrm{n}$ 'future' points have a modal value that is not zero, i.e.

$$
\begin{aligned}
& \left.\left.\operatorname{Mode}\left(P_{(x, y-n)}{ }^{\prime}, P_{(x, y-(n-1))}\right)^{\prime}, \ldots P_{(x, y-1)}{ }^{\prime}, P_{(x, y)}\right)^{\prime}\right)=0 \\
& \operatorname{Mode}\left(P_{(x, y)}{ }^{\prime}, P_{(x, y+1))}{ }^{\prime}, \ldots P_{(x, y+(n-1))}, P_{(x, y+n)}{ }^{\prime}\right) \neq 0
\end{aligned}
$$

To best find this point, $\mathrm{n}$ was set to equal 10 . With this size of inspection width it is possible that the two conditions stated in (3) will be met in the following 9 pixels after P1 because only two 'previous' pixels need to be zero for the modal value to be zero. To over come this problem, only the first occurrence of the conditions being met was noted.

To find P2 the local maximum point on the differentiated surface was found, i.e.

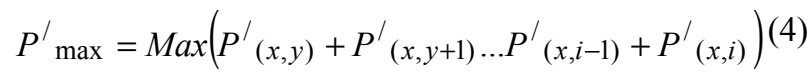

Where $\mathrm{i}$ is the height of the inspection area.

To improve the efficiency of finding P1 and P2 only the top third of the image was inspected because the semiconducting layer was located towards the top of the image, i.e.

$$
\begin{aligned}
& \text { for P1 } y \leq \frac{h}{3}, \text { and } \\
& \text { for P } 2 i=\frac{h}{3}
\end{aligned}
$$

where $h$ is the height of the image

P3 is the point where the surface changes from positive to negative, i.e.

$$
\begin{aligned}
& P^{\prime}(x, y-1) \geq 0 \\
& P^{\prime}(x, y)<0
\end{aligned}
$$

$\mathrm{P} 4$ is similar to P1 in that it is a local maximum and so can be found using (3), however, like P1 and P2, P3 and P4 are located within a certain area of the image. For P3 and P4 this area is the bottom third of the image. This means

$$
\begin{aligned}
& \text { for } \mathrm{P} 3 \frac{2 h}{3} \leq y \leq h, \text { and } \\
& \text { for P } 2 \frac{2 h}{3} \leq y \text { and } i=h
\end{aligned}
$$

\section{Displaying the Results}

The above theory can be used to find P1-4 in a single column of the image. If each column is processed in this way the locations of P1-4 can be found for the whole image. This means that the location of the air to outer semiconductive layer, outer semiconductive layer to insulation, insulation to inner semiconducting layer, and semiconducting layer to conductor interfaces can be found for the whole image. These interfaces can then be displayed by marking every point found on a binary image. In this image the interfaces are marked as ones and the rest of the image as zeros. To improve the binary image created, the interface lines were smoothed to reduce the effects of noise, quantization problems and pixelation. This smoothing does not affect the results and it is assumed that due to the thickness and widths of the tapes used to insulate the joint, local microscopic variations are unlikely to occur. To smooth the lines the vertical location of 10 line pixels, L, either side of each line pixel were found and the median value calculated to produce the new line pixel values, $L^{\prime}$, i.e.

$$
L_{(x, y)}^{\prime}=\operatorname{Median}\left(\begin{array}{c}
L_{(x-10, y)}+L_{(x-9, y)}+\ldots+L_{(x, y)}+\ldots \\
\ldots+L_{(x+9, y)}+L_{(x+10, y)}
\end{array}\right)
$$

This image can be used in two ways. The first method is to highlight the interfaces on the original image. The binary image can be superimposed onto the original joint. In doing this it is easier to see the interface lines in the original image. The second use of the binary image is to calculate the thickness of the semiconducting sheaths and the insulation. The number of pixels between P1 and P2, P2 and P3 and P3 and P4 in each image column can also be counted using a simple incrementor. The number of pixels between each set of points can then be multiplied by the pixel size to determine the thickness of the outer semiconducting sheath, the insulation, and the inner semiconducting sheath. The minimum, average and maximum thicknesses can then be calculated.

\section{RESULTS AND DISCUSSION}

Figure 2(a-c) shows the initial joint images. These three images were then combined to produce Figure 2(d). In Figure 2(a-d) it is possible to see the outer semiconducting sheath and the air above it, however the internal structure of the joint is not visible. So that the internal structure can bee seen the initial histograms of the images in Figure 2(a-d) were shifted towards the white end of the greyscale range to brighten the image for visual purposes, Figure 2(e-h). In these images it is possible to see the ' $\mathrm{H}$ ' shaped defect. The combined image, Figure 2(d), was then processed to create the binary interface line image Figure 2(j). The thickness of the lines has been increased for visualization. This binary image was then superimposed back onto the initial image Figure 2(d) and the shifted histogram image Figure 2(e) to create Figure 2(i) and Figure 2(k) respectively. From these two images it can be seen that all four interfaces have been found, even when the ' $\mathrm{H}$ ' shaped wire defect was occluding the interfaces. The thicknesses of the semiconducting sheaths and the insulation were also calculated and the results, in millimetres are displayed in Table 1. 


\begin{tabular}{|l|c|c|c|}
\hline & Min & Average & Max \\
\hline Outer Semiconducting Sheath & 0.94 & 1.28 & 1.51 \\
\hline Insulation & 14.27 & 14.49 & 14.69 \\
\hline Inner Semiconducting Sheath & 1.38 & 1.51 & 1.56 \\
\hline
\end{tabular}

Table 1 - Calculated Component Thicknesses

To verify these thicknesses the thickness of the insulation and semiconducting sheaths were measured at the end of the joint section using a digital vernier. These are displayed in Table 2.

\begin{tabular}{|l|c|}
\hline Outer Semiconducting Sheath & 0.9 \\
\hline Insulation & 14.4 \\
\hline Inner Semiconducting Sheath & 1.5 \\
\hline
\end{tabular}

Table 2 - Measured Component Thicknesses

The thicknesses measured all fall within the range of values calculated, verifying that the measurements calculated are correct. This point is strengthened further by the fact that in Figure 2(j) the minimum outer semiconducting sheath thickness seems to be located at the end of the joint section, implying that the outer semiconducting sheath at this point should measure approximately $0.9 \mathrm{~mm}$.

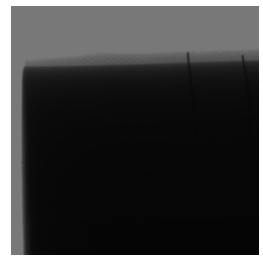

(a)

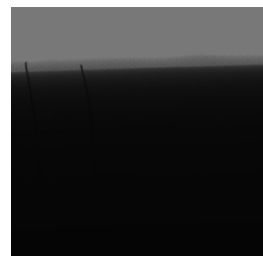

(b)

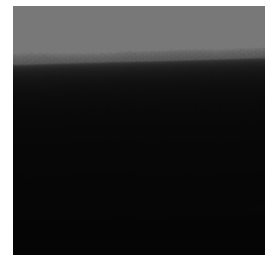

(c)

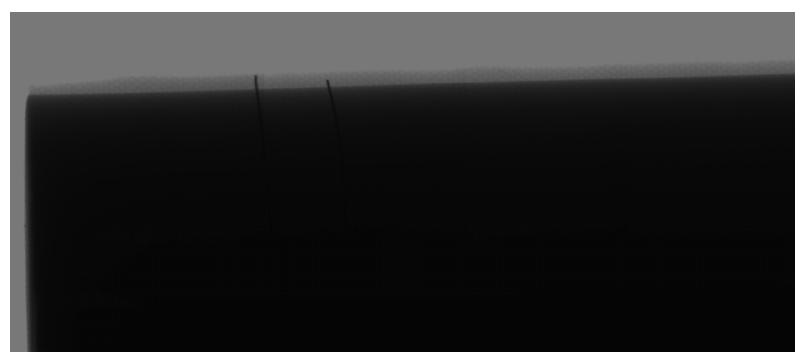

(d)

\section{CONCLUSIONS}

Using the proposed processing technique it is possible to convert the initial image into a binary interface image. The lines in this image denote the boundaries between the conductor, inner semiconducting sheath, the insulation and the outer semiconducting sheath. With this image it is possible to calculate the thicknesses of these components. This data can then be used as a measure of the quality of the joint.

\section{ACKNOWLEDGMENT}

The financial support from National Grid Transco for the initial phase of this project is gratefully acknowledged

\section{REFERENCES}

1. Rowlands, J. A. "Current Advances and Future Trends in X-Ray Digital Detectors", IEEE Instrumentation and Measurement Magazine, Vol. 1 No. 4, 1998, pp. 26-8.

2. Wonnacott, R. J., Wonnacott, T. H. Introductory Statistics, John Wiley \& Sons, 1985, pp. 32.

3. Nixon, M., Aguado, A. Feature Extraction and Image Processing, Oxford, Newnes, 2002, pp. 90-1.

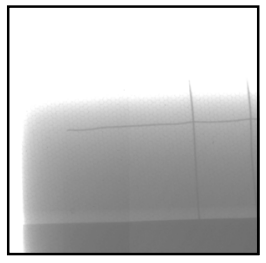

(e)

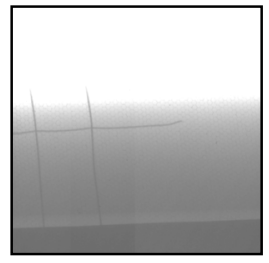

(f)

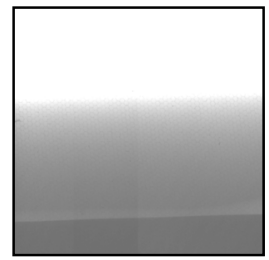

(g)

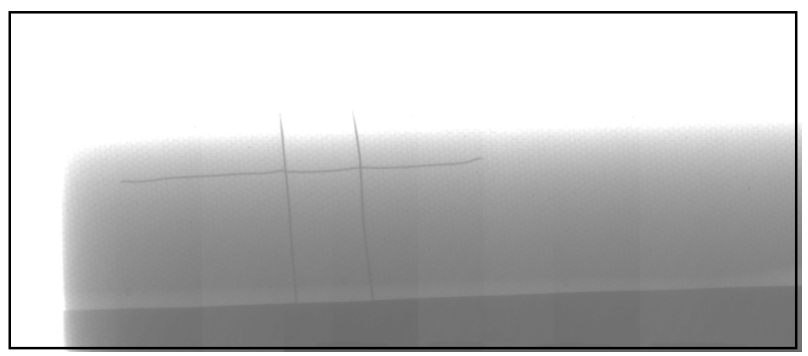

(h)

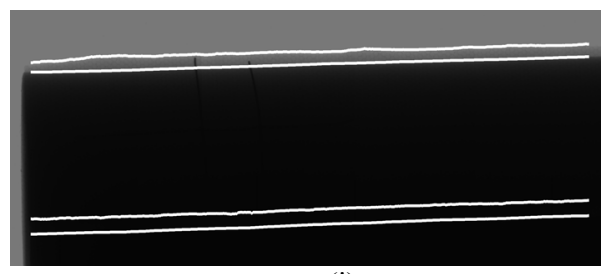

(i)

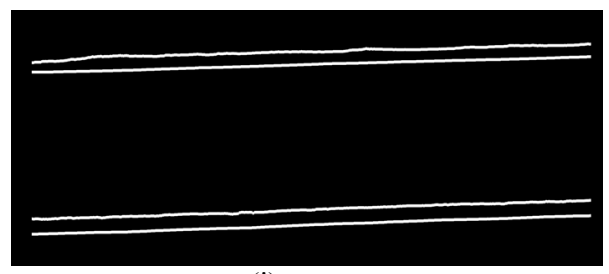

(j)

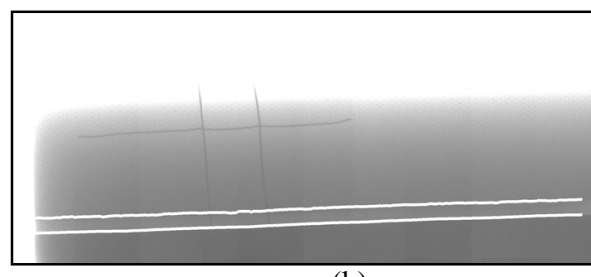

(k)
Figure 2 - (a-c) The original images, (d) the combined images from (a-c), (e-g) The original images with the image histogram shifted to brighten the image in order to show conductor, inner semiconducting sheath and the introduced wire ' $\mathrm{H}$ ' defect, (h) the combined image from (e-g), (i) the interface lines (j) superimposed onto (d), (k) the interface lines (j) superimposed onto (h). 